\title{
LA ACUMULACION TEMPORAL DE SECRETARIAS DE ADMINISTRACION LOCAL, EN ORDEN A LA FUNCION CERTIFICANTE Y ASESORA DEL SECRETARIO
}

352.075 .33

por

\section{Alejandro de Iriarte y Pérez}

Secretario de primera categoría de Administración Local. Diplomado. Secretario General del Ayuntamiento de Móstoles (Madrid).

SUMARIO: I. ASPECTOS GENERALES DEL PROBLEMA: 1. SECRETARf́as VACANTES: aCUMULACIÓN GENERAL. 2. SECRETARÍAS CUBIERTAS: aCUMULACIÓN ACCIDENTAL POR DESCANSO, ENFERMEDAD O EXCEDENCIA TEMPORAL DEL TITULAR.-II. LA ACUMULACION GENERAL DE SECRETARIAS VACANTES: 1. NeCESIDAd DE SU REGUlaCióN: El aUtomatisMo. 2. Obligatoriedad de su aCePtación: A) Por parte de las Corporaciones implicadas. B) Por parte del funcionario. 3. CRITERIos PARA LA DESIGNACIÓN: A) La categoría de la Secretaría. B) La distancia. 4. RÉgimen de la acumulación: A) Dedicación. Horarios. B) Sueldo y dietas. Gratificación complementaria. C) Organización del trabajo. Funciones.-III. LA ACUMULACION ACCIDENTAL: 1. LA DESIGNACIÓN. 2. Su duRación y alCance funcional. 3. Dedicación. 4. Régimen REtriBUTIVO.-IV. CONCLUSIONES.

\section{ASPECTOS GENERALES DEL PROBLEMA}

Es preocupación inveteradamente sentida de los Secretarios de Administración local el hecho - con tristeza, reiteradamente repetido- de que, al producirse la baja del titular de una Secretaría, éste pueda ser sustituido, al menos en la práctica y de la forma más despreocupada, por cualquier funcionario de la Entidad ajeno al 
Cuerpo -incluso alguaciles-, con flagrante olvido de los sagrados deberes que incumben al Secretario: la función fedataria o certificante, para cuyo ejercicio el Secretario se ha perfeccionado a través de un minucioso proceso selectivo, y la asesora, que si bien puede desempeñarse por persona ajena al cargo si se halla debidamente capacitada, debe corresponder al Secretario, como miembro integrante de la Corporación a la que sirve y responsable de su actuación legal.

Hora es ya, hoy que nos encontramos en los umbrales del nacimiento de una nueva normativa de Régimen local, de que en la misma se recoja, de manera efectiva, tan prioritaria cuestión, cuyo olvido y anárquica regulación deja a los Secretarios de Administración local en lugar poco airoso y al ejercicio de su carrera en manos de cualquier funcionario local sin titulación adecuada y sin responsabilidad.

Se hace preciso, sin demora, el estudio cuidadoso y exhaustivo del tema de las acumulaciones de las Secretarías vacantes al objeto de que a las Entidades locales sólo y exclusivamente (y en cualquier momento) pueda asesorarlas, dando fe de sus actos y acuerdos, el funcionario especialmente preparado y capacitado para ello: el Secretario de Administración local. Mantener la anarquía que ha venido imperando hasta, la fecha en torno a este problema presupone admitir la no necesidad de una carrera secretarial - ya que cualquiera puede ejercerla, asesorando y certificando- y la humillación profesional de quienes, con justo título, desempeñan el Secretariado de Administración local.

Afortunadamente, ya la redacción del artículo 68.2 del Real Decreto 3.046/1977, de 6 de octubre, recoge esta vieja aspiración del Cuerpo secretarial -extensivo a los restantes Cuerpos nacionales-, al disponer con rotunda claridad que "las funciones que se les atribuyen sólo podrán ser ejercidas por quienes pertenezcan a los mismos en sus distintas clases...", abandonando la ominosa redacción del artículo 343.4 de la Ley de Régimen local, que preveía la habilitación circunstancial de personas aptas y de reconocida probidad, no pertenecientes a los Cuerpos nacionales, para el ejercicio de sus funciones en Municipios de población inferior a los 500 habitantes.

Del mismo modo, debe considerarse derogado el inciso final del artículo 202 del Reglamento de Funcionarios que hacía referencia a la posibilidad —arriba señalada- de habilitación de un funcio- 
nario debidamente capacitado o incluso de un vecino que, a ser posible, ostentase el título adecuado, a falta de funcionarios interinos o acumulados que pudieren ejercer la Secretaría vacante.

Sin embargo, esta buena intención no es suficiente, ya que, transcurridos casi dos años de la promulgación del referido Real Decreto, las cosas se mantienen en idéntica situación y las Secretarías de Administración local siguen ejerciéndose, con la mayor alegría y despreocupación de los responsables, por personas ajenas a la carrera. Y no se piense que esto ocurre sólo en Ayuntamientos rurales (lo cual, en cualquier caso, no sería excusa con referencia a la función fedataria) de escasa entidad problemática, organizativa y funcional, sino que alcanza a Corporaciones de verdadera importancia demográfica, económica y administrativa.

A tres se reducen las causas que originan la vacancia de las Secretarías de Administración local:

1. ${ }^{2} \quad$ El hecho, claramente perceptible, de ser manifiestamente superior el número de Secretarías que el de Secretarios disponibles para cubrirlas, lo que motiva que casi un 50 por 100 de plazas (si no más) se encuentren crónicamente vacantes, debido a su escaso atractivo profesional. En efecto, en la Provincia de Madrid (por citar un ejemplo quizá no demasiado representativo, debido al alto grado de atractivo de la capital nacional) existen unos ciento ochenta Municipios, para los cuales no pasan de ochenta los profesionales colegiados. Se podría argumentar que el problema apuntado sería corregible mediante el simple hecho de incrementar el número de plazas, pero tal solución no debe ser tan simple cuando después de tantos años aún no se ha alcanzado. Hay que admitir, pues, la existencia de una vacancia crónica o estructural que afecta a determinadas plazas y que, en principio, sólo puede ser cubierta mediante un sistema idóneo de acumulaciones.

2. El mecanismo de los concursos que regulan los traslados, unido a una exagerada lentitud en la tramitación de los mismos, produce igualmente otro tipo de vacancia coyuntural que puede, en ocasiones, prolongarse por dos o más años. Si la plaza es suficientemente atractiva, quizá pueda cubrirse interinamente, pero de no darse esta circunstancia transcurrirá, con toda seguridad, un lapso de tiempo alarmante, que podría enlazar con la vacancia estructural si la plaza presenta escasos alicientes.

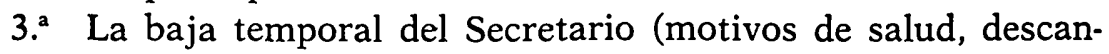
so anual, licencia, permisos) origina un tipo especial de vacancia 
que, si no de derecho, sí de hecho, provoca el desasistimiento de la Secretaría, con consecuencias semejantes a las reseñadas en los apartados anteriores. Cierto es que de tratarse de una baja muy breve (tres o cuatro días) no existe problema, pues raro será el asunto, por complicado y urgente que se presente, cuya resolución no pueda demorarse por este lapso; pero cuando el permiso o la enfermedad se prolongan se produce, de hecho, idéntico problema al planteado en los apartados antecedentes.

Sobre la base de la anterior clasificación, dos son, fundamentalmente, las cuestiones que encierra el tema de las Secretarías vacantes: de una parte, la necesidad de regular la vacancia general provocada por los motivos expuestos en los párrafos referentes a las causas $1 .^{\mathrm{a}}$ y $2 .^{\mathrm{a}}$, regulación que debe venir predeterminada con exactitud y concreción, en aras de la eficaz operatividad del mecanismo; de otra, la menos penosa, pero igualmente grave, causada por el simple hecho del desasistimiento temporal del Secretario por motivos accidentales, cuando en la Corporación no exista sustituto adecuado perteneciente al Cuerpo nacional.

Vamos a tratar por separado ambos aspectos:

\section{Secretarfas vacantes: acumulación General}

Damos siempre por hecho que no existe posibilidad de efectuar nombramiento interino - solución que sería la ideal, tanto desde el punto de vista del funcionario como de la Corporación- para la plaza que ha quedado vacante. Su única posibilidad consiste en acumularla a un Secretario ejerciente en Corporación cercana. No debemos ignorar que existen menos funcionarios que plazas a cubrir.

Este tipo de acumulación que hemos dado en llamar «acumulación general» consiste simplemente en el hecho de tener previsto y regulado automáticamente el mecanismo de la sustitución. Este debe operar en forma inmediata y su posibilidad debe estar reconocida y reglamentada. Vacante cualquier Secretaría de Administración local, en el justo momento en que la misma se produce, e incluso con anterioridad, debe conocerse ya el nombre del funcionario encargado de su acumulación. Para ello deben establecerse los criterios que analizamos en el epígrafe siguiente. 
2. SECRETARÍAs CUBIERTAS: ACUMULACIÓN ACCIDENTAL POR DESCANSO, ENFERMEDAD O EXCEDENCIA TEMPORAL DEL TITULAR

El simple hecho de la baja temporal del Secretario por motivos accidentales, sin llegar a producirse vacante en la Secretaría, debe igualmente estar previsto y regulado. No es admisible que, ausente el Secretario de la Corporación, siquiera sea por muy breve lapso de tiempo, la función certificante la ejerza un funcionario no perteneciente al Cuerpo nacional. Esto representaría, del mismo modo, un fraude a la carrera secretarial. Debe, pues, arbitrarse el mecanismo de sustitución del Secretario por otro Secretario aun en estos supuestos, de la misma forma que a un Notario o a un Médico sólo pueden sustituirles en el ejercicio de sus funciones otro Notario u otro Médico cuando se hallen ausentes, y nunca un oficial de Notaría o un ayudante de clínica.

\section{LA ACUMULACION GENERAL DE SECRETARIAS VACANTES}

\section{LA NECESIDAD DE SU REGULACIÓN: EL AUTOMATISMO}

Se hace necesario regular al detalle el mecanismo de la acumulación general, al objeto de dotar al sistema de la necesaria operatividad. La acumulación de Secretarías existe actualmente como poder en manos de tres fuerzas diferentes: el Gobernador civil, que la decreta; las Corporaciones locales implicadas, que pueden aceptarla o rechazarla; el propio funcionario, que, igualmente, no está obligado a su aceptación. Pues bien, lo que aquí propugnamos es, exactamente, la anulación de las tres fuerzas intervinientes en el proceso de la acumulación, de modo tal que ésta venga predeterminada reglamentariamente y opere en forma automática en el momento de producirse la necesidad. Esto es: que se establezca su obligatoriedad para la autoridad que la decreta o refrenda, para las Corporaciones locales implicadas en el proceso y para el Secretario interesado.

Sólo de esta forma se hará verdaderamente operativo el sistema de acumulaciones. En los momentos actuales, para llegar a la acumulación de una Secretaría pasamos por un proceso que com- 
prende: solicitud de la Corporación interesada al Gobierno civil, acompañada del acuerdo de aceptación de la Entidad implicada y conformidad del Secretario afectado; informe favorable del Colegio Oficial de Secretarios, Interventores y Depositarios de la Provincia de que se trate, y resolución del Gobernador civil. Todo ello puede suponer una demora de dos meses.

A lo que hay que llegar es, sencillamente, a que todo este proceso se halle, de antemano, previsto y regulado reglamentariamente. El automatismo, a nuestro juicio, debe, pues, consistir en lo siguiente:

a) Cada plaza de Secretario debe tener asignadas una serie de plazas acumulables para el caso de que se produzca vacante o ésta exista previamente, de tal forma que todo concursante conozca con antelación a cualquier concurso cuáles sean las Secretarías que a la plaza a la que opta correspondan, caso de hallarse vacantes, y por su correspondiente orden.

b) La asignación de plazas acumulables se regirá teniendo en cuenta los sistemas a que se alude en el epígrafe 3 de este trabajo.

c) Una vez producida la vacante de la primera plaza acumulable, el Secretario a quien ésta corresponda deberá hacerse cargo automáticamente de dicha plaza, salvo excusa grave a discernir por la Dirección General de Administración Local, organismo a quien creemos debe corresponder la organización de los sistemas de acumulación.

Claro está que para la eficacia del sistema propugnado se hace ineludiblemente necesario la confección y el mantenimiento de lo que podríamos llamar "listas» de plazas acumulables, de forma que las mismas se encuentren permanentemente actualizadas. Dicha labor podría ser encomendada a los Colegios Profesionales de Secretarios, Interventores y Depositarios de Administración Local, que, por cierto, tanto han abogado por una regulación como la que defendemos. Al respecto, sería necesario que a cada Corporación se asignasen, al menos, cinco plazas acumulables por orden, según la categoría y la distancia, criterios que estimamos deben presidir la preferencia de las acumulaciones, según exponemos más adelante.

Igualmente deberá tenerse presente el número de acumulaciones que un Secretario puede desempeñar con efectividad y eficacia. Ello estará, lógicamente, en función de la importancia de las mis- 
mas, pero parece aconsejable no se deba exceder de dos acumulaciones, preferentemente si una de ellas pertenece a la primera categoría. En casos de grave necesidad y tratándose de plazas de inferiores categorías podrían acumularse hasta tres plazas o tal vez más.

\section{OBLIGATORIEDAD DE SU ACEPTACIÓN}

Una vez determinadas previamente, por procedimiento reglamentario, las plazas acumulables correspondientes a cada plaza en propiedad, excusado es afirmar la obligatoriedad de la aceptación, ya que, si así no fuera, perdería su fuerza el automatismo que propugnamos. No se nos oculta que esta obligatoriedad puede engendrar cargas pesadas en algunos casos, tanto para las Corporaciones afectadas como para el funcionario, pero entendemos que éste es el único sistema posible para hacer operativa y funcional nuestra Administración local, al menos en su aspecto profesional. Todos los cargos deben llevar cargas, y si deseamos dignificar y honorabilizar nuestra carrera tenemos que aceptar que así sea.

La obligatoriedad que estudiamos tiene dos vertientes:

\section{A) Por parte de las Corporaciones implicadas}

La Corporación cuya Secretaría vaque sabrá ya de antemano quién ha de ser el Secretario que habrá de ejercer la acumulación y vendrá obligada a su aceptación, salvo en el caso, debidamente acreditado y comprobado, de auténtica causa grave de incompatibilidad, que habrá de determinarse por la Dirección General de Administración Local. En este caso, el Secretario a quien correspondiere en primer lugar la acumulación será sustituido por el segundo, y así sucesivamente. Durante el período de comprobación de la causa de incompatibilidad del funcionario, y al objeto de cubrir el posible período de vacancia, ejercerá la acumulación el Secretario siguiente en la lista que fuere aceptado por la Corporación.

\section{B) Por parte del funcionario}

El Secretario a quien reglamentariamente corresponda, una vez producida la vacante, vendrá obligado a posesionarse de inmediato 
de la plaza, dando cuenta del hecho a la Dirección General de Administración Local. Dicha dación de cuenta será siempre obligación del Secretario posesionante.

Del mismo modo que la Corporación, podrá el Secretario excusarse del ejercicio de la acumulación por motivo grave de enfermedad o cualesquiera otros motivos de incompatibilidad demostrados y determinados por la Dirección General de Administración Local. En este caso será sustituido, de manera automática, por quien le siguiere en la lista de Secretarios acumulables.

\section{CRITERIOS PARA LA DESIGNACIÓN}

Es evidente que, previamente a la determinación de las «listas» de plazas acumulables, deberá procederse con el mayor cuidado y objetividad, a fin de no provocar perjuicios al Cuerpo secretarial y a las Corporaciones afectadas, a la confección de las mismas mediante la aplicación de criterios de selección cuidadosamente estudiados. Dos son, a nuestro entender, los que preferentemente deben ser tomados en consideración:

\section{A) La categoria de la Secretaría}

Estando el Cuerpo de Secretarios de Administración Local dividido en tres categorías, para el acceso a las cuales se exigen titulación y pruebas de capacidad diferentes, resulta lógico aceptar que el primer criterio a establecer para la determinación del orden de la acumulación debe ser el de la categoría de la Secretaría. No sería profesionalmente honesto ni conveniente confeccionar la lista de acumulaciones despreciando este criterio, lo que llevaría a la conclusión de que Secretarías de primera categoría, de gran complejidad, pudieran ser ejercidas por Secretarios de tercera que careciesen del título adecuado. No olvidemos que, junto con la función certificante, compete $-\mathrm{y}$ muy fundamentalmente por ciertoal Secretario la función asesora. Es éste extremo que en forma alguna puede ignorarse.

En consecuencia de lo expuesto, podemos dejar sentado lo siguiente:

a) Las Secretarías de primera categoría sólo podrán ser acumuladas por Secretarios de primera (el orden de preferencia podría ser: Secretarios Generales, Vicesecretarios, Oficiales Mayores), y 
sólo en el caso de no existir funcionario alguno de esta categoría en plaza suficientemente próxima (según criterio siguiente) para no hacer inoperante la acumulación, por Secretarios de segunda, licenciados en Derecho o en Ciencias Políticas.

b) Las Secretarías de segunda categoría podrían ser acumuladas, indistintamente (según criterio de cercanía o proximidad), por Secretarios de primera o segunda categorías, éstos, preferentemente, licenciados en Derecho o Ciencias Políticas. Sólo en el caso de no hallarse disponibles Secretarios de estas categorías podrían acumularse a Secretarios de tercera.

c) Las Secretarías de tercera categoría podrían ser acumuladas a Secretarios de las tres categorías indistintamente y según criterio de proximidad.

Justifica esta postura, que podría ser criticada por algunos Secretarios de segunda o tercera categorías, el hecho del mayor número de plazas vacantes en estas dos categorías que en la primera, así como el plano de desigualdad en que se encontrarían los Secretarios de primera, si sólo les fueren permitidas acumulaciones dentro de su propia categoría, dado el menor número de vacantes en ésta. La observación de la realidad nos da la razón si contemplamos el gran número de Secretarios de tercera con tres o cuatro plazas acumuladas, mientras muchos de primera no ostentan ninguna.

En cualquier caso no se debe olvidar que la mejor preparación de los primeros les hace plenamente capaces para el ejercicio de cualquier Secretaría.

\section{B) La distancia}

Determinado el criterio funcional, que debe ser prioritario, fijaremos nuestra atención, al tiempo de establecer las listas de plazas acumulables, en la distancia a la plaza vacante y que ha de ejercerse por acumulación. Pese a que hoy el criterio de la distancia ha perdido la significación que tuvo en el pasado, debido al alto desarrollo de los medios de comunicación, no debe desdeñarse la "comodidad" que supone la cercanía, que ha de permitir al funcionario, aparte de los desplazamientos reglamentarios impuestos, mantener un contacto más activo con la Corporación acumulada a la que sirve, en casos de necesidad o urgencia. En cualquier su- 
puesto, parece más racional distribuir las acumulaciones tomando en consideración la menor distancia a la plaza titular.

Apuntado lo anterior y sentada la racionalidad del criterio de la distancia, no encontraremos excesivos obstáculos para la acumulación de Secretarías fuera de la propia Provincia, cuando se encuentren suficientemente próximas a la plaza titular, ya que entendemos que el régimen de acumulaciones debe organizarse con ámbito nacional y sancionarse por la Dirección General de Administración Local.

\section{Régimen de la acumulación}

Una vez sentados los fundamentos jurídicos de la acumulación sobre las bases del automatismo y la obligatoriedad en la aceptación, sería necesario fijar las condiciones mínimas y vinculantes sobre las que dicha acumulación debe descansar inexcusablemente: los derechos y deberes que conlleva el régimen de acumulación, según la categoría, la importancia de la plaza y la dedicación que se estime necesaria.

\section{A) Dedicación. Horarios}

En la regulación normativa que se establezca para las acumulaciones debe determinarse la dedicación semanal que tal plaza reclame, teniendo en cuenta su categoría, problemática y complejidad del trabajo a desarrollar. Es claro que la dedicación no ha de ser la misma para una Corporación de 50.000 habitantes, por ejemplo, que para una de 1.000 .

De menor a mayor, estimamos que un criterio de posible aplicación podría ser el siguiente:

a) Secretarias de tercera categoria: ocho horas semanales, que podrían distribuirse en dos tardes, con lo que se estaría capacitado para la atención de hasta tres Secretarías acumuladas. Para el caso de que se estimase necesaria una dedicación mayor, podría llegarse al establecimiento de un horario superior, compensado mediante la correspondiente gratificación complementaria.

b) Secretarias de segunda categoría: doce horas semanales, distribuidas a lo largo de tres tardes o de una mañana y una tarde. Con esto serían susceptibles de acumulación dos plazas de esta ca- 
tegoría. Si se estimase necesaria una dedicación superior, ésta sería compensada mediante gratificación complementaria.

e) Secretarias de primera categoria: veinte horas semanales, distribuidas a lo largo de una mañana y tres tardes, con el establecimiento de la misma gratificación complementaria para el supuesto de una superior dedicación. Con estos horarios no sería posible la acumulación de más de una Secretaría de primera, aunque sí cabría la posibilidad de acumular, además, otra de segunda o de tercera.

Estas dedicaciones, que se estiman mínimas, deberían ser conocidas por las respectivas Corporaciones, de tal modo que, al momento de producirse las acumulaciones, no pudieran presentarse objeciones por parte de aquéllas. De igual modo y a los mismos efectos deberán ser conocidas por los Secretarios afectados.

Es de la máxima importancia resaltar que la acumulación de plazas por los Secretarios de Administración local es un derecho y un deber inherente a la función de los mismos. Que se trata de un auxilio profesional a la Administración local y que en forma alguna puede considerarse como gabela o pluriempleo de los funcionarios que la ejercen.

\section{B) Sueldo y dietas. Gratificación complementaria}

Si se pretende una dedicación permanente y efectiva, como la apuntada en el epígrafe anterior, debe, lógicamente, establecerse un régimen de retribuciones digno y suficiente. No olvidemos que, además, lo que se pretende es hacer obligatoria la acumulación para todo Secretario y que, en consecuencia, en la mayor parte de los casos representará un auténtico sacrificio.

En la fijación de este tipo de remuneraciones debemos distinguir tres conceptos, claramente diferenciados.

a) Sueldo. Es la compensación económica que debe cifrarse al funcionario por el desempeño de su gestión secretarial, por el trabajo realizado y por la responsabilidad asumida. En consecuencia, estimamos debe abarcar el 100 por 100 de los emolumentos de la plaza; esto es, sueldo básico, complemento de destino y grado, así como pagas extraordinarias. Deben quedar fuera de este concepto los aumentos graduales (ya que las plazas acumuladas no deben generar antigüedad), incentivos, prolongaciones de jornadas 
(salvo lo referente a gratificación complementaria, que estudiaremos después), gratificaciones por servicios extraordinarios y especiales y ayuda familiar.

b) Dietas. Debe abarcar la compensación por gastos de desplazamiento y otros extraordinarios (comidas, etc.) y podría cifrarse tomando en consideración lo establecido por la legislación general en esta materia, o bien teniendo en cuenta el kilometraje, las facilidades de comunicación, la duración de la jornada y, por supuesto, el número de desplazamientos efectuados mensualmente. Sería conveniente la determinación de un tanto alzado que se percibiría mensualmente junto con el sueldo.

c) Gratificación complementaria. Unicamente se percibirá para el caso de que la Corporación cuya Secretaria se acumule estime necesaria una mayor dedicación a la establecida reglamentariamente, y podría cifrarse sobre la base del exceso de horas que el funcionario venga obligado a trabajar por encima de su dedicación normal.

En cualquier caso, debe señalarse que la jornada superior a que se alude en el párrafo anterior no debe producir detrimento a la Corporación de la que el Secretario es titular, por lo que dicho exceso deberá cubrirse obligatoriamente fuera de la jornada de trabajo que tuviere establecida la Corporación titular y sin perjuicio para la misma.

\section{C) Organización del trabajo. Funciones}

Resulta claro que las funciones a desempeñar por un Secretario en régimen de acumulación no pueden abarcar en modo alguno la amplitud que alcanzan cuando se es titular de la plaza, con plena dedicación. La diferencia horaria ya es clara: de cuarenta y dos horas en esta última, a veinte en la primera, en el supuesto más favorable. Esto lleva como consecuencia el que al Secretario acumulado deben recortársele funciones, entendiendo que lo que la Corporación cuya Secretaría se acumula precisa es un director de la gestión jurídico-administrativa y nunca funcionarios técnicos, administrativos o auxiliares con los que, a tenor de su categoría, debe contar.

En principio nosotros nos ceñiríamos al título del presente trabajo y colocaríamos como obligaciones prioritarias del Secretario 
acumulado aquellas que hacen estricta referencia a las funciones certificante y asesora, a las que, de jure, se extiende su responsabilidad. De acuerdo a esto, deberán ser funciones inexcusables las siguientes:

a) Asistir, con voz deliberante, a todas las sesiones que celebren el Pleno de la Corporación y la Comisión Municipal Permanente, donde existiere.

b) Redactar el acta de las sesiones mencionadas (debe entenderse responsabilizarse de su redacción) y encargarse de su transcripción al libro correspondiente.

c) Certificar de todos los actos o resoluciones de la Presidencia y de los acuerdos adoptados por el Pleno y Comisión Municipal Permanente, así como de los libros y documentos de la Entidad.

d) Autorizar y dar fe de todas las licitaciones, contratos y actos en general en que intervengan la Corporación o su Presidente.

e) Advertir, dentro de la esfera de su competencia, de toda manifiesta ilegalidad en las resoluciones y acuerdos que se pretendan adoptar.

f) Informar en las sesiones sobre los antecedentes y el aspecto legal de los asuntos que lo requieran.

g) Asesorar a la Corporación y a su Presidencia, emitiendo dictámenes cuando éstas lo dispongan o el asunto, a su juicio, revista la debida relevancia.

h) Acompañar al Presidente en los actos de firma de escrituras o documentos de importancia y, cuando aquél lo precise, en sus visitas a autoridades y asistencia a reuniones.

Por el contrario, no estimamos en absoluto procedente el ejercicio de sus facultades como jefe de personal (de las que debe relevársele), ya que, dada su especial dedicación, en gran parte fuera de los horarios normales de oficina, su contacto con el personal es tangencial, perdiendo forzosamente efectividad su posible control o vigilancia, siendo, por otra parté, perfectamente posible el ejercicio de esta función por cualquier otro funcionario especialmente delegado y capacitado para ello, sin menoscabo alguno de la auténtica gestión secretarial.

Sí podría ejercer las funciones como coordinador de las dependencias y servicios de la Corporación, ya que dicha función no implica, en líneas generales, una dedicación habitual y constante, pu- 
diendo desempeñarse sin estricta sujeción a horarios concretos cuando ello no menoscabare sus funciones primordiales.

En cuanto a la función directiva de la Secretaría General, sí podría encargarse de la alta gestión organizativa, dictando, cuando lo estimare conveniente, circulares para el más correcto funcionamiento de la Secretaría, delegando en el funcionario que tenga a su cargo la Unidad Administrativa de Asuntos Generales, la inspección de dicha función organizativa, a la que, en general, se hace referencia en el artículo 145 del Reglamento de Funcionarios. Obvio resulta que lo expuesto anteriormente sólo parece aplicable cuando se trate de Corporaciones de una gran complejidad funcional.

En cualquier caso, se considera de la mayor transcendencia el que se maticen al detalle las funciones del Secretario acumulado, toda vez que, debido a sus limitaciones horarias, no resulta en absoluto posible abarcar la amplísima gama a que hacen referencia los artículos 141 a 145 del Reglamento de Funcionarios, debiendo ceñirse a aquellas funciones que por su índole estrictamente secretarial le competen irrenunciablemente. Pretender lo contrario sería hacer perder operatividad y eficacia a la gestión del funcionario acumulado.

\section{LA ACUMULACION ACCIDENTAL}

Hemos dicho al comienzo que el tipo de vacancia producido por la ausencia temporal del titular de una Secretaría, aunque menos penoso, resultaba igualmente grave. Permítasenos corregir: consideramos de la más alta gravedad este tipo de vacancia accidental, que viene dando origen a los mayores y más graves abusos, en menoscabo de la función secretarial. La acumulación general de Secretarías vacantes al menos existe actualmente, aunque escasa y anárquicamente regulada. Para el tipo de vacancia que ahora estudiamos no existe previsto en la legislación actual mecanismo alguno de suplencia o acumulación, lo que da origen a las más sangrantes arbitrariedades a la hora de suplir al Secretario ausente. En efecto, normalmente éste viene siendo despreocupadamente sustituido por cualquier funcionario de la Corporación, incluso auxiliares y hasta alguaciles, sin preparación ni responsabilidad alguna.

La justificación de esta vacancia accidental (aparte lo expuesto en el párrafo precedente) viene determinada por el hecho de que durante la ausencia del titular de la Secretaría puede efectivamente 
presentarse el problema de difícil solución, el asunto de gravedad que no admite demora, etc. En algunas Corporaciones, carentes de personal especializado, se produciría, de hecho, un paro si tales problemas no pueden ser resueltos sobre la marcha. Muchos profesionales de la Administración local hemos comprobado cómo Presidentes de algunas Corporaciones, en ausencia del Secretario, nos han consultado, incluso telefónicamente, sobre tal o cual materia de difícil solución. Esto no debe ocurrir, no debería ocurrir, si se hallase prevista la acumulación accidental o sustitución del Secretario titular, incluso para períodos de días.

Las dos justificaciones que acabamos de exponer representan motivo más que suficiente para que se arbitre el procedimiento adecuado sustitutorio: la primera paliaría el más flagrante ataque contra la profesión secretarial, impidiendo - como ya muy bien recoge la normativa vigente (art. 68.2 del Real Decreto 3.046/1977, de 6 de octubre citado) - que personas ajenas al Cuerpo nacional puedan ejercer funciones que competen exclusivamente a éste. La segunda cubriría, para las Corporaciones temporalmente desasistidas, el período de baja del Secretario, evitando así las posibles paralizaciones en el desenvolvimiento de su gestión.

Dado que en estos casos, al existir titular de la Corporación (sólo se trata, como hemos expuesto, de supuestos de enfermedad -inferior a seis meses-, vacaciones anuales, licencias, etc.), no se hace precisa una dedicación tan intensa, resulta obvio que la regulación de este sistema debe revestir características especiales, que pasamos a continuación a considerar.

\section{LA DESIGNACIÓN}

Debe estar igualmente prevista previamente mediante un cuadro de sustituciones, correspondiendo en todo caso al funcionario titular la comunicación al sustituto de su cese temporal y sin que en ningún caso pueda autorizarse dicho cese sin que éste se haya hecho cargo de la Secretaría. Entendemos que para este tipo de acumulación accidental de la plaza — podría llamarse sustitución temporal-, dadas sus especiales características de brevedad y excepcionalidad, debe bastar el simple Decreto de la Alcaldía o acuerdo municipal, al que debe acompañarse siempre el visto bueno del Secretario titular, dando cuenta a la Dirección General de Administración Local. 
$\mathrm{Su}$ cese, en consecuencia con las propias características de la designación, debe venir simplemente determinado por el alta del funcionario titular, con comunicación escrita al Secretario acumulado accidentalmente, a las Corporaciones afectadas y a la Dirección General.

\section{SU DURACIÓN Y ALCANCE FUNCIONAL}

La duración de la sustitución viene lógicamente determinada por la de la ausencia del funcionario titular. Sin embargo, debemos advertir que para el caso de enfermedad que se prolongue por más de seis meses deberá arbitrarse lo necesario para establecer el sistma de acumulación general, de conformidad con la situación a la que pase el funcionario titular.

No existe, por supuesto, inconveniente alguno para que las sustituciones que aquí estudiamos puedan repetirse tantas veces fuere necesario, de acuerdo a la propia naturaleza del mecanismo sustitutorio.

Hemos señalado al inicio de este epígrafe que el alcance funcional de este tipo de acumulación o sustitución temporal, por su propia naturaleza, no puede extenderse a la dimensión que se ha dado a la acumulación general en la que el Secretario acumulado es el auténtico director de la gestión administrativa de la Secretaría, al no existir Secretario titular. En ésta, el Secretario acumulado sólo «sustituye» temporalmente a un funcionario titular de la Secretaría y no resulta admisible que asuma funciones que puedan suponer intromisión en la dirección secretarial, correspondiente por derecho al propietario.

Creemos que sus funciones deben ceñirse estrictamente a las de certificar de los actos y acuerdos corporativos y a las de asesoramiento. Por las primeras deberá asistir a las sesiones del Pleno y de la Comisión Permanente, con observación de los deberes inherentes a las mismas. Por las segundas prestará su asesoramiento a la Corporación, así como a su Presidente y Comisiones.

\section{DEDICACIÓN}

En consecuencia con el alcance de sus funciones, al Secretario sustituto podrá bastarle con una dedicación superficial, sin que previamente pueda ésta determinarse, ya que dependerá en cada 
caso de las necesidades surgidas. Indudablemente deberá, al menos, señalarse dos o tres horas semanales, en jornada de trabajo, para la firma y despacho de los documentos que lo requieran, así como para el conveniente cambio de impresiones con las autoridades o funcionarios de la Corporación.

\section{RÉGIMEN RETRIBUTIVO}

Dependerá igualmente de la intensidad de la dedicación, pudiendo, en principio, establecerse en un 50 por 100 del sueldo, según criterios señalados para la acumulación general y con percibo de las dietas que pudieren corresponderle por los traslados que realice.

\section{CONCLUSIONES}

Para concretar, finalmente, las opiniones vertidas en el presente trabajo, podemos resumir las siguientes conclusiones:

1. Necesidad de una regulación normativa sobre el tema de las acumulaciones de plazas de Cuerpos nacionales, basada en el reconocimiento de que las funciones encomendadas a los mismos sólo pueden ser ejercidas por quienes pertenezcan a los indicados Cuerpos, como ya reconoce el Real Decreto 3.046/1977, de 6 de octubre.

2. $\quad$ El régimen de acumulaciones de plazas debe descansar sobre un automatismo reglamentariamente establecido, mediante la determinación de cuadros o listas de plazas acumulables para cada Corporación, al objeto de que, vacante una Secretaría, su acumulación se produzca, por el funcionario a quien corresponda, de forma instantánea.

3. ${ }^{\mathrm{a}} \quad$ Establecer la obligatoriedad de la acumulación, tanto para las Corporaciones afectadas como para los funcionarios implicados.

4. Al regular la acumulación con carácter de ámbito nacional, las competencias que hoy corresponden al Gobierno Civil deben ser asumidas por el Director General de Administración Local, quien refrendará las designaciones automáticamente establecidas, una vez comunicada la vacante por el Presidente de la Corporación y la aceptación por los interesados, visará los cuadros o lis- 
tas confeccionados por los Colegios profesionales y resolverá cuantas cuestiones pudieren suscitarse incidentalmente.

5. Se regularán con precisión la dedicación, alcance funcional y retribución de la acumulación.

6. $\quad$ No debe olvidarse el importante papel que debería corresponder a los Colegios profesionales, los cuales deberán asumir la responsabilidad de confeccionar y mantener constantemente actualizados los cuadros o listas de funcionarios en situación de acumulables, en orden a la correcta operatividad del sistema.

7. Aunque hemos ceñido nuestro análisis a las funciones del Secretario de Administración local, resulta claro que lo expuesto puede y debe tener idéntica aplicación para los Interventores e incluso para los Depositarios, ya que las funciones que los mismos desempeñan no pueden ser abandonadas en manos de profanos, ajenos a los Cuerpos nacionales. Cada uno de estos funcionarios, dentro de su respectiva esfera de competencia, ejerce la función asesora y la jefatura de los servicios que le están encomendados. En este sentido, no puede caber duda de su derecho y de su obligación a la acumulación de plazas vacantes.

Por último, debemos advertir que las opiniones que en el presente análisis vertemos no tienen otra pretensión ni otro alcance que el lanzamiento de ideas sobre las cuales podría descansar una futura regulación del tema de las acumulaciones de Secretarías vacantes (igualmente aplicable a Intervenciones y Depositarías), que tanta trascendencia encierra, especialmente en orden a evitar o paliar en lo posible los efectos negativos del intrusismo actualmente imperante en la carrera secretarial y del menoscabo del prestigio de sus profesionales. Al hallarse este campo hoy prácticamente virgen de la más somera atención, son válidas cuantas ideas propendan a la determinación del sistema idóneo y han de ser bien venidas, en cuanto tiendan a eliminar la actual situación de desorden que se padece en la materia. Esta es la exclusiva pretensión del autor de estas líneas. 


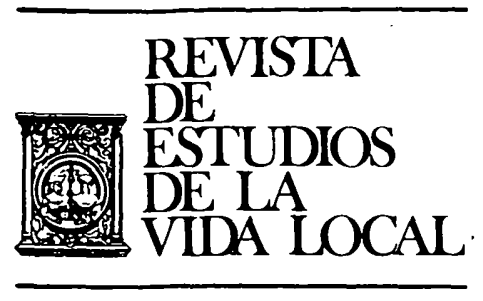

\section{CRONICAS}


REVL-1979, núm. 204. IRIARTE Y PEREZ, ALEJANDRO DE. LA ACUMULACION TEMPORAL DE S...

REVL-1979, núm. 204. IRIARTE Y PEREZ, ALEJANDRO DE. LA ACUMULACION TEMPORAL DE S... 